\title{
Gender and secondary risk assessment following an ST-elevation myocardial infarction
}

This article was published in the following Dove Press journal:

Nursing: Research and Reviews

16 August 2013

Number of times this article has been viewed

Elizabeth Scruth ${ }^{1,3}$

Linda Worrall-Carter ${ }^{\prime}$

Eugene Cheng ${ }^{2}$

'St Vincent's/ACU Centre for Nursing Research, School of Nursing and Midwifery, Faculty of Health Sciences, Australian Catholic University, Melbourne, VIC, Australia; ${ }^{2}$ Kaiser Permanente Medical Group, San Jose, CA, USA; ${ }^{3}$ Kaiser Permanente Northern California, Oakland, CA, USA
Purpose: The Thrombolysis in Myocardial Infarction (TIMI) risk score, Global Register of Acute Coronary Events (GRACE) risk score, and the Controlled Abciximab and Device Investigation to Lower Late Angioplasty Complications (CADILLAC) risk score are validated predictors of secondary events and death after an acute coronary syndrome (ACS). In our study, we sought to examine the predictability of the TIMI, GRACE, and the CADILLAC risk scores in women undergoing percutaneous coronary intervention for ST-elevation myocardial infarction (STEMI) for in-hospital, 1-year major cardiac events, nonmajor cardiac events, and mortality. A limited number of studies examining the secondary risk scores for use after STEMI in women have been conducted. Most studies have been conducted in both men and women without separating out the predictability in women in regard to the various risk scores.

Patients and methods: In a subanalysis of women from a larger study of both men and women with STEMI, a 1-year follow up of 77 women with STEMI was undertaken using a retrospective approach and comparing the TIMI, GRACE, and CADILLAC risk scores for in-hospital and 1-year outcomes of major cardiac events, nonmajor cardiac events, and death. The predictive value of the models was assessed with evaluation of the area under the curve in receiver operating-characteristic analysis.

Results: The study revealed that risk stratification of female patients with STEMI early after presentation using the TIMI risk score or after angiography using the CADILLAC risk score may provide important prognostic information and enable accurate identification of high-risk patients.

Conclusion: Though limited by sample size and retrospective analysis, our study provided evidence into the validity of using existing secondary risk tools in women. Further studies are needed to determine the risk score that is most predictive for women presenting with STEMI and treated with percutaneous coronary intervention. It may be useful to incorporate the risk scores into clinical practice to guide short- and long-term follow-up after STEMI in women as a preventive strategy.

Keywords: cardiovascular risk, acute coronary syndromes, nonmajor cardiac events, major cardiac events

\section{Introduction}

A quick and accurate method of diagnosis of acute coronary syndrome - ST-elevation myocardial infarction (ACS-STEMI) is essential to determine the type of invasive strategy required. Percutaneous coronary intervention (PCI) is the evidence-based treatment for acute STEMI. ${ }^{1}$ There were 622,000 PCI procedures performed in the US in 2007 (of which 360,000 were on women), while in 2006 the average cost per patient was $\$ 48,399 .{ }^{2,3}$ Nearly $43 \%$ of patients with ACS are women. ${ }^{3}$ The widespread
Correspondence: Elizabeth Scruth Kaiser Permanente Northern California, 1950 Franklin Street

Oakland, CA 94612, USA

$\mathrm{Tel}+\mathrm{I} 5 \mathrm{I} 09874475$

Email Elizabeth.Scruth@kp.org 
adoption of PCI for STEMI has led to decreases in mortality and morbidity. ${ }^{4,5}$ Even with the standard treatment for STEMI being PCI, variability in survival will exist due to variables such as patient comorbidities and delay in seeking treatment. Risk assessment at the time of the STEMI for in-hospital events and death and during follow-up after discharge will assist the clinician to engage in preventive strategies to lessen risks that may occur at later time intervals. Risks associated with PCI are highest within the first 24-48 hours after the procedure and include periprocedural myocardial infarction, acute stent thrombosis, bleeding, or renal failure. ${ }^{6,7}$ The American Heart Association (AHA) and the American College of Cardiology (ACC) recommend risk stratification in all patients presenting with STEMI.

Several risk tools for assessing death and cardiovascular events after ACS have been developed. The risk tools were developed using both demographic and electrocardiographic components. ${ }^{7-12}$ The Thrombolysis in Myocardial Infarction (TIMI) risk score for STEMI was initially derived from a set of patients treated with fibrinolysis agents and validated in an external data set from the TIMI 9 trial. ${ }^{13}$ The TIMI risk score for STEMI patients was further validated in an unselected population of patients with STEMI treated with fibrinolysis therapy, and showed a strong predictive value $(C=0.79) .{ }^{11}$ In 2005, the Controlled Abciximab and Device Investigation to Lower Late Angioplasty Complications (CADILLAC) risk score was developed. ${ }^{12}$ As with the TIMI risk tool, the CADILLAC risk score was based on randomized trials of patients. The CADILLAC risk score was validated using external data sets from other PCI trials. Predictions for 30-day and 1-year mortality rates after primary PCI for ACS using the CADILLAC risk score revealed $C$-statistics of 0.83 and 0.81 for 30 -day mortality and 0.79 and 0.78 for 1 -year mortality. ${ }^{12}$ The Global Register of Acute Coronary Events (GRACE) score was developed differently from the CADILLAC and TIMI risk scores. It was based on a large registry of patients who experienced all types of ACS, and thus was not limited to STEMI. The GRACE risk score incorporated similar components to the TIMI risk score: clinical and electrocardiographic characteristics used to determine risk. The GRACE risk score was shown to be of predictive value for all forms of ACS. ${ }^{13,14}$ Table 1 outlines the development of the CADILLAC, GRACE, and TIMI risk tools and their components.

Comparisons of the risk scores in ACS patients have been reported in the literature. ${ }^{15,16}$ The aim of this study was to do a subanalysis of the performance of the TIMI, GRACE, and CADILLAC risk scores in a cohort of women who had been part of a larger study involving men, and determine if the risk tools remained valid and sensitive. ${ }^{17}$

The risk of cardiovascular disease in women as they age is well documented in the literature, and it is only within the last 5 years that the health-care profession has recognized the risk. ${ }^{18}$ Women are still underrepresented in clinical trials, with only a $5 \%$ increase from 1990 to $2000 .{ }^{19}$ Women with STEMI present with worse clinical profiles than their male counterparts, leading to increased rates of mortality and cardiac events in hospital and in the long term after PCI. ${ }^{19}$

Table I GRACE, TIMI, and CADILLAC risk tools: development and components

\begin{tabular}{|c|c|c|c|}
\hline $\begin{array}{l}\text { Risk tool and predictive } \\
\text { accuracy (C-statistic) }\end{array}$ & Population derived from & $\begin{array}{l}\text { Primary end } \\
\text { points }\end{array}$ & Components of the risk tool \\
\hline $\begin{array}{l}\text { GRACE } \\
0.47 I-0.533\end{array}$ & $\begin{array}{l}\text { Acute coronary syndrome } \\
\text { patient population }\end{array}$ & $\begin{array}{l}\text { Hospital mortality } \\
\text { 6-month mortality }\end{array}$ & $\begin{array}{l}\text { Inclusion: (only tool to include cardiac arrest during presentation) } \\
\text { Parameters: age, Killip class, systolic blood pressure, ST deviation, } \\
\text { cardiac arrest during presentation, serum creatinine concentration, } \\
\text { elevated serum cardiac biomarkers, heart rate }\end{array}$ \\
\hline $\begin{array}{l}\text { TIMI } \\
0.6 \mathrm{I}-0.724\end{array}$ & $\begin{array}{l}\text { Highly selected acute } \\
\text { coronary syndrome } \\
\text { patients treated with } \\
\text { fibrinolysis }\end{array}$ & $\begin{array}{l}\text { Hospital mortality } \\
\text { 30-day mortality }\end{array}$ & $\begin{array}{l}\text { Inclusion: (only tool to include weight and evidence of left-bundle } \\
\text { branch block and anterior myocardial infarction-specific area } \\
\text { of infarction) } \\
\text { Parameters: age, diabetes mellitus, angina, hypertension, systolic } \\
\text { blood pressure }<100 \mathrm{mmHg} \text {, heart rate }<100 \text { beats/minute, Killip } \\
\text { class II-IV, weight }<65 \mathrm{~kg} \text {, anterior myocardial infarction or left- } \\
\text { bundle branch block, time to treatment }>4 \text { hours }\end{array}$ \\
\hline $\begin{array}{l}\text { CADILLAC } \\
0.685-0.824\end{array}$ & $\begin{array}{l}\text { Based on individuals with } \\
\text { acute coronary syndrome } \\
\text { treated invasively }\end{array}$ & $\begin{array}{l}\text { Hospital mortality } \\
\text { 30-day mortality } \\
\text { I-year mortality }\end{array}$ & $\begin{array}{l}\text { Inclusion: (only tool to include anemia, TIMI flow, left ventricular } \\
\text { ejection fraction, and evidence of triple-vessel disease) } \\
\text { Parameters: left ventricular ejection fraction }<40 \% \text {, Killip } \\
\text { class II-III, renal insufficiency, TIMI flow grade after PCl of 0-2, } \\
\text { age }>65 \text { years, anemia, triple-vessel disease }\end{array}$ \\
\hline
\end{tabular}

Note: Data from ${ }^{14,15}$

Abbreviations: GRACE, Global Register of Acute Coronary Events; TIMI, Thrombolysis in Myocardial Infarction; CADILLAC, Controlled Abciximab and Device Investigation to Lower Late Angioplasty Complications; PCI, percutaneous coronary intervention. 
The clinical factors thought to contribute to a worse clinical profile in females with ACS (including STEMI) include delayed onset of disease (8-10 years later than men), older age at presentation, smaller body surface area, and more comorbidities, including diabetes mellitus (DM) ${ }^{19}$ In a review of over 70,000 medical records of patients presenting with their first acute myocardial infarction, women with DM aged below 65 years had poorer outcomes than their male counterparts. ${ }^{20}$ In a study comparing adverse outcomes after contemporary PCI in women versus men with ACS, DM, cholesterol, and type of lesion were predictors of major adverse cardiac events in women, whereas peripheral arterial disease, age, current smoking, left anterior descending coronary artery lesion, and baseline hematocrit were the strongest predictors of major adverse cardiac events in men. ${ }^{19}$ The AHA reports $65 \%$ of women who die suddenly from cardiovascular disease display no symptoms, making risk assessment both from a primary aspect and a secondary aspect important in women. ${ }^{3}$ None of the risk-assessment tools used to assess risk after PCI are sex-based, and they do not currently address the variability in long-term risk.

\section{Methods}

\section{Research design/sample and data collection}

A retrospective subanalysis of 109 women was carried out in Northern California Kaiser Permanente medical centers and affiliated facilities for women admitted with a STEMI diagnosis identified by International Classification of Diseases version 9 codes from 2007 to 2008. The women were part of a study including men ( $\mathrm{n}=186$ patients; 109 [57\%] men, age $60.05 \pm 10.93$ years; 77 [41\%] women, age $67.38+11.75$ years) previously published. ${ }^{17}$ The dates included were January 2007 to December 2008, with follow-up to December 2009. Permission for the study was granted through the Division of Research of Northern California Kaiser Permanente institutional review board. Only data from complete health-care records were included in the analysis. The STEMI diagnosis required to confirm patients' symptoms consistent with an ACS have been published previously. ${ }^{17}$ These were consistent with the standard definition of ACS-STEMI at the time of the study. ${ }^{1}$

Eligible patients were female adults 18 years of age or older meeting the above criteria for STEMI. Exclusion criteria consisted of women presenting in cardiogenic shock (to closely replicate the original patient population selections in the development and validation of the riskassessment tools).
The data for calculating the risk scores were collected directly from the medical record. In-hospital data included prehospital drug history, admission electrocardiography reports, blood results, diagnostic tests and medical events, medical interventions, and discharge summary that included any events not captured previously. The post-PCI discharge data included total mortality, cardiac mortality, noncardiac mortality, confirmed myocardial infarction, interventional cardiology treatment ( $>30$ days after discharge), cardiothoracic surgery treatment ( $>30$ days after discharge), incidence of stroke, and cardiac/noncardiac hospitalization. Total mortality was used as the main end point for our study, with causes of death obtained from the medical record.

All women in our study were treated for ACS-STEMI according to the hospital management guidelines for chest pain and STEMI, based on the ACC/AHA Guidelines for the Management of Patients with STEMI at the time of their presentation, which included the interventional pathway for acute STEMI as a default treatment. ${ }^{1}$ Pharmacological treatment was optimized according to the current guidelines for STEMI. Coronary angiography and angioplasty (PCI) was performed on all the women.

The women were treated with aspirin $325 \mathrm{mg}$ before PCI and clopidogrel $600 \mathrm{mg}$ administered either before the PCI or immediately after. Unfractionated heparin $(70 \mathrm{U} / \mathrm{kg}$ loading) was administered before the PCI. Selection of stent type was determined by the interventional cardiologist treating the patient. Patients were prescribed lifelong aspirin and clopidogrel (75 mg/day) for 3-12 months upon discharge. The three risk scores were calculated from the initial history, electrocardiograph, laboratory values and coronary angiogram (Table 1). The study was retrospective, with all data collected entered in a computer database. In-hospital events and events at 12 months were recorded from the electronic hospital records. Repeated revascularization procedures and episodes of reinfarction were collected from the electronic medical record, as well as all data from contracted hospitals from scanned-in documents.

\section{Results}

Data analysis was conducted utilizing the receiver operatingcharacteristic curve and $C$-static methodology. Statistical analysis was performed using SAS software 9.1 for logistic regression and 9.2 for the receiver operating-characteristic curve analysis (SAS Institute, Cary, NC, USA). A total of 77 (from 109) (those with incomplete medical records [25\%] were excluded acknowledging a possible bias in the analysis) with STEMI were included in the final study. 
Baseline characteristics and medications used by the women at time of admission for the STEMI are listed in Table 2. In-hospital major cardiac events (MCEs) included retroperitoneal bleeding, cardiogenic shock, fatal myocardial infarction, and revascularization. During the hospitalization period of the women, there was one death and two non-MCEs (NMCEs). Follow-up at 1 year revealed there were no MCEs and two NMCEs (Table 3).

Area under the curve ( $C$-statistic) of the three risk scores and comparisons for all events and deaths were conducted, and the risk scores with the highest performance are listed in Table 4. The TIMI and CADILLAC risk scores performed well for inhospital mortality ( 0.860 and 0.853 ) compared to the GRACE $C$-statistic of 0.645. For in-hospital MCEs, the CADILLAC risk score performed better than the TIMI and GRACE, with a $C$-statistic of 0.718 compared to 0.632 and 0.680 . All three risk scores were comparable for in-hospital NMCEs, with $C$-statistics of 0.642 for the CADILLAC risk score, 0.624 for the GRACE risk score, and 0.668 for the TIMI risk score, indicating that none was superior in predictability of events.

For 1-year mortality, the CADILLAC risk score and the TIMI risk score performed better, with $C$-statistics of 0.806 and 0.7663 compared to the GRACE risk score, with a $C$-statistic of 0.645 . When comparing the three risk scores for performance for prediction of NMCEs at 1 year, the CADILLAC and TIMI risk scores, with $C$-statistics of 0.619 and 0.604 , respectively, performed slightly better than the

Table 2 Demographics and cardiac medications on admission

\begin{tabular}{ll}
\hline Patient demographics $(\mathbf{n}=\mathbf{7 7})$ & \\
\hline Age (years) & $67.38 \pm$ II.75 \\
Diabetes mellitus & $22(28.57 \%)$ \\
Hypertension & $54(70.13 \%)$ \\
Hyperlipidemia & $52(67.53 \%)$ \\
Smoking (current) & $20(33.90 \%)$ \\
Previous myocardial infarction & $5(6.49 \%)$ \\
Previous medications & \\
$\quad$ Aspirin & $43(55.84 \%)$ \\
Clopidogrel & $8(10.39 \%)$ \\
$\quad$ Beta-blockers & $49(63.64 \%)$ \\
$\quad$ Statins & $54(70.13 \%)$ \\
$\quad$ Angiotensin-converting enzyme inhibitors & $35(45.45 \%)$ \\
$\quad$ Angiotensin-receptor blockers & $5(6.58 \%)$ \\
On admission & \\
Heart rate (beats/minute) & $75.16 \pm 16.35$ \\
Systolic blood pressure $(\mathrm{mmHg})$ & $124.09 \pm 27.92$ \\
Diastolic blood pressure $(\mathrm{mmHg})$ & $68.32 \pm 15.55$ \\
Killip's classification $>$ I & $36(46.75 \%)$ \\
Left ventricular ejection fraction $<40 \%$ & $12(16.00 \%)$ \\
Anterior wall myocardial infarction & $28(36.36 \%)$ \\
Inferior wall myocardial infarction & $47(61.04 \%)$ \\
Lateral wall myocardial infarction & $2(2.60 \%)$ \\
\hline
\end{tabular}

Table 3 Events: hospital, I-year follow-up, death $(n=77)$

\begin{tabular}{lll}
\hline Event & Hospital & I-year follow-up \\
\hline MCE & II & 0 \\
NMCE & 2 & 2 \\
Death & 1 & 2 \\
\hline
\end{tabular}

Abbreviations: MCE, major cardiac event; NMCE, nonmajor cardiac event.

GRACE risk score, with a $C$-statistic of 0.547 . A comparison of the scores for any in-hospital event and any event in 1 year were also analyzed using the same methodology and a binary logic model with Fisher's scoring-optimization technique. The results revealed the CADILLAC risk score may be more predictive. Figures 1 and 2 illustrate the results.

\section{Discussion}

The present study, based on a small sample (from a larger study including 109 men published in 2012) from a multihospital system of female patients with a diagnosis of STEMI and treated using PCI, compared the predictive accuracy of three risk scores for clinical outcomes in hospital, at 1 year, and death. We found that the TIMI and CADILLAC risk scores performed better for in-hospital and 1-year mortality. For in-hospital MCEs, the CADILLAC performed better than the GRACE and TIMI risk tools. All three risk tools had relatively similar predictive values in the $0.6-0.668$ range for NMCEs in hospital and at 1 year, with the CADILLAC and TIMI performing slightly better than the GRACE risk score for NMCEs at 1 year. When comparing any events in hospital and at 1 year, the CADILLAC risk score performed slightly better than the TIMI and GRACE risk scores.

Comparison of our study to that reported in the literature reveals similar results, although not separated out for women. Méndez-Eirín et al reported in a comparison of four common risk-prediction scores that TIMI, CADILLAC, and GRACE all had high predictive accuracy for determination of risk after PCI for ACS. They did not separate out the women from the men. ${ }^{21}$ A study examining STEMI patients compared the same four risk-prediction tools as Méndez-Eirín et al revealed CADILLAC was superior to TIMI and GRACE in men and women undergoing PCI for STEMI. ${ }^{14}$

Table 4 Comparison of risk-tool performance

\begin{tabular}{lll}
\hline Event & Hospital (C-statistic) & I-year (C-statistic) follow up \\
\hline MCE & CADILLAC 0.718 & No events \\
NMCE & TIMI 0.660 & CADILLAC 0.610 \\
Death & CADILLAC 0.860 & CADILLAC 0.80 \\
\hline
\end{tabular}

Abbreviations: MCE, major cardiac event; NMCE, nonmajor cardiac event; TIMI, Thrombolysis in Myocardial Infarction; CADILLAC, Controlled Abciximab and Device Investigation to Lower Late Angioplasty Complications. 


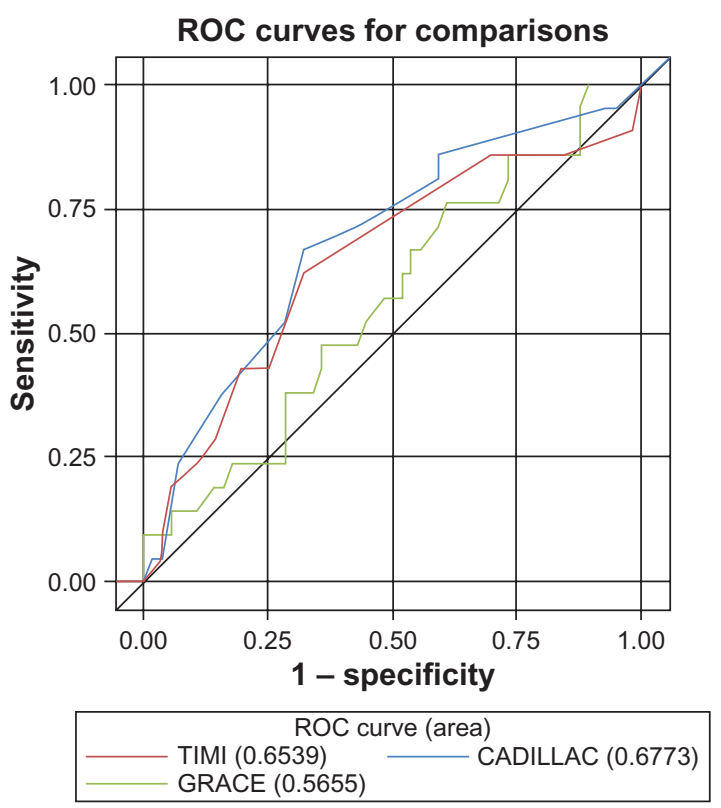

Figure I Any event (MCE/NMCE) and/or death in hospital.

Abbreviations: MCE, major cardiac event; NMCE, nonmajor cardiac event; GRACE, Global Register of Acute Coronary Events; TIMI, Thrombolysis in Myocardial Infarction; CADILLAC, Controlled Abciximab and Device Investigation to Lower Late Angioplasty Complications; ROC, receiver operating characteristic.

We used three contemporary risk scores in our study. Comparison of the composition of the risk tools used in the present study revealed that all included components of age and Killip's classification were shown to be important predictors of survival after STEMI in the literature. ${ }^{12,13}$

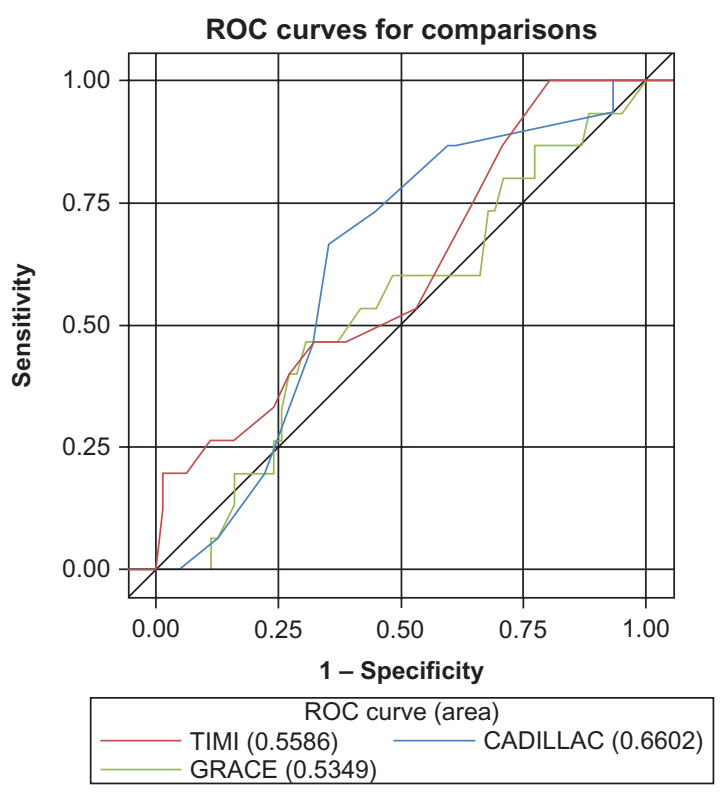

Figure 2 Any event (MCE/NMCE) and/or death at I year.

Abbreviations: MCE, major cardiac event; NMCE, nonmajor cardiac event; GRACE, Global Register of Acute Coronary Events; TIMI, Thrombolysis in Myocardial Infarction; CADILLAC, Controlled Abciximab and Device Investigation to Lower Late Angioplasty Complications; ROC, receiver operating characteristic.
The CADILLAC risk tool was inherently different from the TIMI and GRACE risk tools, as it included the angiographic parameters of triple-vessel disease and final blood flow in the infarcted artery, as well as left ventricular ejection fraction. Therefore, the CADILLAC risk score can only be calculated after interpretation of angiographic results and PCI, which may limit its use to patients treated using an invasive approach. Baseline left ventricular ejection fraction has been reported in the literature to be a strong determinant of survival after STEMI. ${ }^{12,22}$ Another unique component of the CADILLAC risk tool was the inclusion of the component anemia, which has emerged as a predictor of mortality after STEMI. ${ }^{23}$ The CADILLAC risk tool, however, is not helpful for risk-stratifying STEMI patients prior to angiography, and the TIMI and GRACE risk tools are more helpful in the early stage, as they do not rely on angiographic parameters. The use of the CADILLAC risk tool therefore may not be useful in determining if the patient requires transfer to another facility for a higher level of care, as it requires data from angiography to complete the risk scoring.

The weak predictive value of the GRACE risk score in our subanalysis of outcomes of women with STEMI treated with PCI may be related to the components of the risk score. ST-segment deviation and increased cardiac enzymes are components included in the GRACE risk score, and all the women presented with these parameters. The parameters are not unique to STEMI, as they are present in the spectrum of ACS. Components that the GRACE risk score does not include are the location of the myocardial infarction, and left ventricular ejection fraction, as it was originally developed in patients who presented with all types of ACS, not just acute myocardial infarctions. The development of the risk tool was therefore not limited to STEMI patients, which could account for the lack of specificity and predictability in the STEMI population.

The authors acknowledge the limitations of the study. The sample size is small, underpowered, and is representative of one health-care system whose members primarily have employer-funded health coverage.

Using risk-assessment tools that have been validated across different sexes, ethnic groups, and age-groups is critical to ensuring the treatment plan and follow-up plan is specific to the patient. Meaningful use of diagnostic imaging and laboratory tests that are specific to the degree of risk assessed using the risk-assessment tools can then be planned utilizing the risk score derived from the patient's data at time intervals during their follow-up. 


\section{Conclusion}

Although limited by sample size and underpowered, the present subanalysis provides an important analysis of women and their risk after an acute myocardial infarction. Given the limited knowledge in the existing literature around these risk scores and women, we have provided an important contribution to global cardiovascular risk assessment in women. Our study reveals that risk stratification of female patients with STEMI early after presentation using the TIMI risk score or after angiography using the CADILLAC risk score provides important prognostic information and may enable accurate identification of high risk. Knowledge of sex-based differences in mortality and in identifying the predictors of long-term outcomes after PCI in ACS with the assistance of secondary risk-prediction tools can help prepare patients and health-care providers for the expected outcomes and events. Therapy at the time of ACS and after intervention should be optimized based on these differences. Estimating risk provides an understanding of the underlying mechanisms of the disease process, and can guide preventive measures to limit future risk of events occurring leading to increased mortality. Risk assessment of women with cardiovascular disease should be a continual process, involving both primary risk assessment from an early age with the use of validated, reliable risk tools, and further secondary risk assessment should occur at the time of a cardiac event with appropriate risk tools. Enrollment of more women in clinical trials and registries is needed to report sex-specific outcomes, as predictors of risk in women will continue to evolve as technology advances and patient risk profiles change. Further studies adequately powered to conduct multivariate risk adjustment with cohorts of ethnically diverse women are needed to determine if the risk scores are most predictive for women presenting with STEMI and treated with PCI. Analysis of the individual components of the risk tools that women most commonly experience during a STEMI is also needed in larger studies.

\section{Acknowledgment}

The authors would like to acknowledge the generous support for the publishing fee from the Northern California Nursing Research Division of Kaiser Permanente; Dr Gretchen Summer, nurse scientist and director.

\section{Disclosure}

The authors report no conflicts of interest in this work.

\section{References}

1. Kushner FG, Hand M, Smith SC, et al. 2009 Focused Updates: ACC/ AHA Guidelines for the Management of Patients with ST-Elevation Myocardial Infarction (updating the 2004 Guideline and 2007 Focused Update) and ACC/AHA/SCAI Guidelines for on Percutaneous Coronary Intervention (Updating the 2005 Guideline and 2007 Focused Update): a report of the American College of Cardiology Foundation/American Heart Association Task Force on Practice Guidelines. Circulation. 2009;120:2271-2306

2. American Heart Association. Heart Disease and Stroke Statistics: 2009 Update. Dallas: American Heart Association; 2009.

3. Roger VL, Go AS, Lloyd-Jones DM, et al. Heart disease and stroke statistics - 2012 update: a report from the AHA. Circulation. 2012;125: 188-197.

4. Keely EC, Boura JA, Grines CL. Primary angioplasty versus intravenous thrombolytic therapy for acute myocardial infarction: a quantitative review of 23 randomized trials. Lancet. 2003;361:13-20.

5. Rao SV, Ohman EM. Anticoagulant therapy for percutaneous coronary intervention. Circ Cardiovasc Interv. 2010;3:80-88.

6. Rao SV, Kaltenbach LA, Weintraub WS, et al. Prevalence and outcomes of same-day discharge after elective percutaneous coronary intervention among older adults. JAMA. 2011;13:1461-1467.

7. Lee KL, Woodlief LH, Topol EJ, et al. Predictors of 30-day mortality in the era of reperfusion for acute myocardial infarction. Results from an international trial of 41,021 patients. GUSTO-I Investigators. Circulation. 1995;91:1659-1668.

8. Fresco C, Sardinia F, Maggioni AP, et al. Very early assessment of risk for in-hospital death among 11,483 patients with acute myocardial infarction. GISSI Investigators. Am Heart J. 1999;138: 1058-1064.

9. Morrow DA, Antman EM, Charlesworth A, et al. TIMI risk score for ST-elevation myocardial infarction: a convenient, bedside, clinical score for risk assessment at presentation: an intravenous nPA for treatment of infarcting myocardium early II trial substudy. Circulation. 2000;102:2031-2037.

10. Morrow DA, Antman EM, Parsons L, et al. Application of the TIMI risk score for ST-elevation MI in the National Registry of Myocardial Infarction 3. JAMA. 2001;286:1356-1359.

11. Halkin A, Singh M, Nikolsky E, et al. Prediction of mortality after primary percutaneous coronary intervention for acute myocardial infarction. The CADILLAC risk score. J Am Coll Cardiol. 2005;45: 1397-1405.

12. Granger CB, Goldberg RJ, Dabbous O, et al. Predictors of hospital mortality in the global registry of acute coronary events. Arch Intern Med. 2003;163:2345-2353.

13. Eagle, KA, Lim MJ, Dabbous $\mathrm{OH}$, et al. A validated prediction model for all forms of acute coronary syndrome: estimating the risk of 6-month postdischarge death in an international registry. JAMA. 2004;291: 2727-2733.

14. Lev EI, Kornowski R, Vaknin-Assa H, et al. Comparison of the predictive value of four different risk scores for outcomes of patients with STelevation acute myocardial infarction undergoing primary percutaneous coronary intervention. Am J Cardiol. 2008;102:6-11.

15. Filipak KJ, Koltowski L, Grabowski M, et al. Prospective comparison of the 5 most popular risk scores in clinical use for unselected patients with acute coronary syndrome. Circ J. 2011;75(1):167-173.

16. Brkovic V, Dobric M, Beleslin B, et al. Additive prognostic value of the SYNTAX score over GRACE, TIMI, ZWOLLE, CADILLAC and PAMI risk scores in patients with acute ST-segment elevation myocardial infarction treated by primary percutaneous coronary intervention. Int J Cardiovasc Imaging. Epub February $27,2013$.

17. Scruth E, Cheng E, Worrall-Carter L. Risk score comparison of outcomes in patients presenting with ST-elevation myocardial infarction treated with percutaneous coronary intervention. Eur J Cardiovasc Nurs. Epub June 13, 2012. 
18. Shaw LJ, Bairey Merz CN, Pepine CJ, et al. Insights from the NHLBI-Sponsored Women's Ischemia Syndrome Evaluation (WISE) study: Part I: gender differences in traditional and novel risk factors, symptom evaluation, and gender-optimized diagnostic strategies. $J$ Am Coll Cardiol. 2006;47:S4-S20.

19. Pendyala LK, Torgoson R, Loh JP, et al. Comparison of adverse outcomes after contemporary percutaneous coronary intervention in women versus men with acute coronary syndrome. Am J Cardiol. 2013;111 1092-1098.

20. Norhammar A, Stenestrand U, Lindbäck J, Wallentin L. Women younger than 65 years with diabetes mellitus are a higher risk group after myocardial infarction: a report from the Swedish Register of Information and Knowledge about Swedish Heart Intensive Care Admission (RIKSHIA). Heart. 2008:94;1565-1570.
21. Méndez-Eirín E, Flores-Ríos X, García-López F, et al. Comparison of the prognostic predictive value of the TIMI, PAMI, CADILLAC and GRACE Risk Scores in STEACS undergoing primary or rescue PCI. Rev Esp Cardiol. 2012;65:227-233.

22. Halkin A, Stone, GW, Dixon SR. Impact and determinants of left ventricular ejection fraction in patients undergoing primary percutaneous coronary intervention in acute coronary syndromes. Am J Cardiol. 2005;96:325-331.

23. Nikolsky E, Aymong ED, Halkin A, et al. Impact of anemia in patients with acute myocardial infarction undergoing percutaneous coronary intervention: analyses from the Controlled Abciximab and Device Investigation to Lower Late Angioplasty Complications (CADILLAC) trial. J Am Coll Cardiol. 2004;44:547-553.

\section{Publish your work in this journal}

Nursing: Research and Reviews is an international, peer-reviewed, open access journal publishing original research, reports, reviews and commentaries on all aspects of nursing and patient care. These include patient education and counselling, ethics, management and organizational issues, diagnostics and prescribing, economics and

\section{Dovepress}

resource management, health outcomes, and improving patient safety in all settings. The manuscript management system is completely online and includes a very quick and fair peer-review system. Visit http://www.dovepress.com/testimonials.php to read real quotes from published authors.

Submit your manuscript here: http://www.dovepress.com/nursing-research-and-reviews-journal 